\title{
INFLUENCE OF RATE DEPENDENT PLASTICITY ON AN SHEET METAL BENDING PROCESS
}

\author{
Christian Reisinger ${ }^{\S *}$, Christian Zehetner ${ }^{\S}$, Hans Irschik ${ }^{\dagger}$, Michael Krommer ${ }^{\dagger}$ and Wolfgang \\ Kunze $^{\mathrm{X}}$ \\ ${ }^{\S}$ University of Applied Sciences Upper Austria \\ Stelzhammerstraße 23, 4600 Wels, Austria \\ e-mail: christian.reisinger@fh-wels.at,web page: https://www.fh-ooe.at \\ †Institute of Technical Mechanics, Johannes Keppler University \\ Altenbergerstraße 69, $4040 \mathrm{Linz}$, Austria \\ e-mail: michael.krommer@jku.at, web page: https://www.jku.at/tmech \\ x Salvagnini Maschinenbau GmbH \\ Dr.-Guido-Salvagnini-Straße 14482 Ennsdorf, Austria \\ Email: wolfgang.kunze@salvagninigroup.com - Web page: http://www.salvagnini.at
}

\begin{abstract}
The high demands on precision and quality of industrial sheet metal forming processes are increasing steadily. Therefore, more and more effects concerning the machines but also the material behaviour of the workpiece must be considered. Here, we consider an automatic panel bender of Salvagnini Maschinenbau $\mathrm{GmbH}$ [1]. In this application, it turned out that the speed of bending is a relevant influence factor.

Goal of this work is to estimate the influence of strain rate on bending forces and the shape of the bent profile. In a previous work [2], an analytic model in the framework of Bernoulli-Euler beam theory for the bending of a cantilever with elasto-plastic behaviour has been presented, dimensionless influence parameters have been derived, and it has been shown that the influence parameters obtained by linear beam theory are also relevant for the nonlinear industrial application involving large deformations. To include the strain rate dependency, the beam model now has been extended to elasto-visco-plastic behaviour. Besides the plastic deformations also the elastic ones are important, since the springback angle must be known exactly to achieve the required accuracy. To represent this effect, Hollomon's equation [3] was extended by an elastic term. Additionally, the strain rate sensitivity is considered according to [4].

With this beam model, numerical studies have been performed, in which forces, and deflections have been analysed for small deformations. Finally, the results of beam theory have been compared with 3D-Finite Element computations. A very good coincidence turned out. The results also show that the strain rate has a significant influence on deformations and forces. The outcome of the present study will be used to consider the strain rate as an additional parameter in the digital twin of the automatic panel bender [1].
\end{abstract}

\section{REFERENCES}

[1] C. Zehetner et al., "High-quality sheet metal production using a model-based adaptive approach," Procedia Comput. Sci., vol. 180, no. 2019, pp. 249-258, 2021.

[2] C. Zehetner, F. Hammelmüller, H. Irschik, and W. Kunze, "Similarity methods in elasto-plastic beam bending," in COMPLAS XIII: proceedings of the XIII International Conference on Computational Plasticity: fundamentals and applications, 2015, pp. 906-915.

[3] H. Hollomon, “Tensile deformation.," Aime Trans, vol. 12, no. (4), pp. 1-22, 1945.

[4] Patrick Larour, "Strain rate sensitivity of automotive sheet steels: influence of plastic strain, strain rate, temperature, microstructure, bake hardening and pre-strain,"

Rheinisch-Westfälische Technische Hochschule Aachen, 2010. 